Revista Brasileira de Agricultura Irrigada v.10, nº.1, p. 420 - 427, 2016

ISSN 1982-7679 (On-line)

Fortaleza, CE, INOVAGRI - http://www.inovagri.org.br

DOI: $10.7127 /$ rbai.v10n100343

Protocolo 343.16 - 07/08/2015 Aprovado em 23/01/2016

\title{
COMPORTAMENTO DAS EQUAÇÕES DE SCOBEY, MANNING E FAIR- WHIPPLE-HSIAO QUANDO UTILIZADAS PARA ESTIMATIVA DA PERDA DE CARGA EM TUBOS COM COMPORTAMENTO ELÁSTICO
}

\author{
Osvaldo Rettore Neto ${ }^{1}$, Vitor Emanuel Quevedo Tavares ${ }^{2}$, Lessandro Coll Faria ${ }^{3}$, Luciana \\ Marini Köpp ${ }^{4}$, Luis Carlos Timm ${ }^{5}$
}

\begin{abstract}
RESUMO
A indústria de plásticos e derivados, com o auxílio da engenharia, tem aprimorado a qualidade do material destinado à fabricação de tubos, principalmente os de polietileno. A elasticidade desses tubos permite que ocorra aumento do diâmetro interno com o aumento da pressão, o que não é levado em consideração pelos equacionamentos matemáticos utilizados para determinação da perda de carga. O objetivo deste trabalho foi verificar o erro na estimativa da perda de carga contínua em tubos elásticos em função da pressão de entrada na linha, comparando valores observados e estimados pelas equações de Scobey, Manning e FairWhipple-Hsiao. Avaliaram-se 3 amostras de tubo de polietileno sob 9 pressões de entrada e 3 vazões, sendo estas correspondentes às velocidades de 1,0; 1,5 e 2,0 $\mathrm{m} \mathrm{s}^{-1}$. Efetuou-se a medição do diâmetro externo dos tubos nas pressões entre 0 a 588,4 kPa, com incrementos de 49,05 kPa. Para tubos com característica elástica, observou-se que as equações não estimaram adequadamente a perda de carga contínua. A diferença encontrada entre os valores calculados e observados utilizando a equação de Manning variou de 36,5\% a 77,3\% para a equação FairWhipple-Hsiao variou de $37,9 \%$ a $55,3 \%$ e para a equação de Scobey variou de $6,1 \%$ a $30,2 \%$.
\end{abstract}

Palavras-chaves: perda de carga contínua, irrigação localizada, tubos de polietileno

\section{BEHAVIOR OF SCOBEY, MANNING AND FAIR-WHIPPLE-HSIAO EQUATIONS WHEN USED TO ESTIMATE THE HEAD LOSS IN ELASTIC PIPES}

\footnotetext{
ABSTRACT

${ }^{1}$ Eng. Agrícola, Prof. Adjunto, Departamento de Engenharia Rural, Faculdade de Agronomia Eliseu Maciel, Universidade Federal de Pelotas, Campus Universitário s/n Capão do Leão - RS, CEP: 96010-610, e-mail: osvaldo.rettore@ufpel.edu.br (autor correspondente)

${ }^{2}$ Eng. Agrícola, Prof. Associado, Departamento de Engenharia Rural, FAEM/UFPel, Capão do Leão - RS, email: veqtavares@yahoo.com.br

${ }^{3}$ Eng. Agrícola, Prof. Adjunto, CDTec/UFPel, Pelotas - RS, e-mail: lessandrofaria@gmail.com

${ }^{4}$ Eng. Agrícola, Prof ${ }^{\mathrm{a}}$. Adjunta, Departamento de Engenharia Rural, FAEM/UFPel, Capão do Leão - RS, e-mail: lucianakopp@gmail.com

${ }^{5}$ Eng. Agrícola, Prof. Associado, Departamento de Engenharia Rural, FAEM/UFPel, Capão do Leão - RS, email: lcartimm@yahoo.com.br
} 
The plastics and derivatives industry, with the help of engineering, has enhanced the quality of the material used to manufacture pipes, especially the polyethylene ones. The elasticity of these pipes allows an increase in its internal diameter with increasing pressure, which is not taken into account by mathematical equations used to determine head loss. In this study we intend to verify the error in the estimation of head loss in elastic pipes considering the inlet pressure in line, comparing observed and estimated values by the equations of Scobey, Manning and Fair-Whipple-Hsiao. Three samples of polyethylene pipe were evaluated under a combination of nine inlet pressures and three water flow rates, corresponding to velocities of 1.0, 1.5 and $2.0 \mathrm{~m} \cdot \mathrm{s}^{-1}$. We conducted the measurement of the external diameter of the pipes under pressures ranging from 0 to $588.4 \mathrm{kPa}$, with $49.05 \mathrm{kPa}$ increments. It was observed that the equations did not adequately estimate the friction head losses for elastic pipes. The differences between calculated and observed values ranged from $36.5 \%$ to $77.3 \%$ for the Manning equation, from $37.9 \%$ to $55.3 \%$ for Fair-Whipple-Hsiao and from $6.1 \%$ to $30.2 \%$ for Scobey.

Keywords: friction head loss, localized irrigation, polyethylene pipes

\section{INTRODUÇÃO}

A agricultura irrigada é hoje o maior consumidor de água no mundo e como a matriz energética brasileira está baseada na produção de energia hidroelétrica, há necessidade de constantes aprimoramentos técnicos e científicos para maximizar o uso da água e racionalizar o consumo energético para aumentar as áreas de produção agrícola com os recursos naturais disponíveis (RETTORE NETO, 2011).

A perda de carga contínua representa a dissipação de energia da água em forma de calor, ao longo da tubulação, decorrente da resistência ao escoamento oferecida pela viscosidade do fluido e pela inércia das partículas, sendo variável com a rugosidade da parede do tubo, diâmetro da tubulação e com a velocidade da água (PORTO, 2006).

Existe um predomínio de material plástico nas tubulações das redes de distribuição das parcelas irrigadas por gotejamento e microaspersão. Segundo Gomes (1997), para tubulações de pequenos diâmetros, que transportam pequenas vazões requeridas nas unidades ou parcelas irrigadas, os tubos de plástico fabricados em polietileno de baixa densidade (PEBD) são economicamente mais competitivos que os tubos dos demais materiais disponíveis no mercado.

Os projetistas de sistemas de irrigação utilizam frequentemente as equações de perda de carga existentes na literatura, considerando os diâmetros internos das tubulações informados pelos fabricantes. Contudo, em razão desses tubos serem produzidos com materiais plásticos, seus diâmetros podem modificar-se em virtude das variações na pressão de operação. Isso poderia influenciar na perda de carga real, o que resultaria em alterações nas condições hidráulicas projetadas (VILELA et al., 2003).

A equação de Manning resultou de uma simplificação da expressão de GanguilletKutter, sendo está formulada em 1867. Evidentemente, no decorrer de tantos anos a indústria dos materiais e técnicas de fabricação dos tubos evoluíram bastante, assim, a superfície interna dos tubos apresenta-se mais homogênea e mais favorável ao escoamento (AZEVEDO NETTO; ALVAREZ, 1992).

Segundo Gomes (1997), a equação de Manning aplica-se a todo tipo de conduto, tanto nos que a água escoa em lâmina livre, como nos escoamentos sob pressão. Está amplamente experimentada, e sempre teve bastante aplicação por conta de sua simplicidade e aceitável precisão dos resultados. Para o caso especifico de tubulações, a equação assume a seguinte forma:

$$
h f=6,36 \frac{n^{2} V^{2}}{D^{1,33}} L
$$


em que: $h f$ - perda de carga contínua na tubulação (m); $n$ - coeficiente de Manning; $V$ velocidade média do escoamento $\left(\mathrm{m} \mathrm{s}^{-1}\right) ; D-$ diâmetro interno do tubo (m); $L$ - comprimento do tubo (m).

Segundo a NBR 5626, a perda de carga ao longo de um tubo depende do seu comprimento e diâmetro interno, da rugosidade da sua superfície interna e da vazão. Para calcular o valor da perda de carga nos tubos, recomenda-se utilizar a equação universal, conhecendo-se os valores das rugosidades. $\mathrm{Na}$ falta dessa informação, pode ser utilizada a expressão de Fair-Whipple-Hsiao indicada a seguir:

Para tubos lisos (tubos de plástico, cobre ou liga de cobre):

$$
j=8,69 \cdot 10^{6} Q^{1,75} D^{-4,75}
$$

em que: $j$ - perda de carga unitária $\left(\mathrm{kPa} \mathrm{m}^{-1}\right) ; Q$ - vazão $\left(\mathrm{l} \mathrm{s}^{-1}\right) ; D$ - diâmetro interno do tubo (mm).

A equação de Scobey é indicada para o cálculo das perdas de carga nas tubulações leves, utilizadas nos sistemas de irrigação por aspersão, e por gotejamento (GOMES, 1997). Sua equação é dada pela seguinte expressão:

$$
h f=\frac{K s}{387} \frac{1}{D^{1,1}} V^{1,9} L
$$

em que: $h f$ - perda de carga contínua na tubulação (m); Ks - coeficiente de Scobey; $V$ velocidade média do escoamento $\left(\mathrm{m} \mathrm{s}^{-1}\right) ; D-$ diâmetro interno do tubo (m); $L$ - comprimento do tubo (m).
O objetivo deste trabalho foi quantificar os erros na determinação da perda de carga em tubos elásticos em função da pressão de entrada na linha, comparando valores observados e estimados pelas equações de Scobey, Manning e Fair-Whipple-Hsiao.

\section{MATERIAL E MÉTODOS}

A pesquisa foi conduzida no Laboratório de Irrigação e Hidráulica do Departamento de Engenharia de Biossistemas - ESALQ/USP, Piracicaba-SP. Montou-se uma bancada de ensaio em circuito hidráulico fechado para pressurização em regime dinâmico do fluído (água), que facilitou o controle, monitoramento e aquisição dos dados necessários para desenvolvimento do trabalho.

Utilizou-se um reservatório com capacidade de $28.000 \mathrm{~L}$ e uma motobomba de 2,0 CV, vazão entre 0 e $5,510^{-4} \mathrm{~m}^{3} \mathrm{~s}^{-1}$ e pressão variando de 0 a 784,6 $\mathrm{kPa}$. No recalque foram instalados dois registros de gaveta e uma válvula reguladora de pressão de diafragma para controlar a pressão de entrada no tubo.

A condução da água do sistema de bombeamento e controle até o início da linha (tubo de polietileno) foi feita por meio de tubos flexíveis de alta pressão com diâmetro de 1". A conexão entre os tubos foi feita por adaptadores internos, abraçadeiras e conexões hidráulicas (luva de união e bucha de redução). Tubos com $40 \mathrm{~m}$ de comprimento, em nível, foram utilizados para determinação da perda de carga contínua. Na Tabela 1 apresentam-se as características técnicas do tubo utilizado.

Tabela 1. Características do tubo ensaiado.

\begin{tabular}{llll}
\hline Fabricante & Diâm. Interno (mm) & Espessura da parede (mm) & Material \\
\hline Plasnova Ind. Com. Ltda. & 15,759 & 0,996 & $\begin{array}{l}\text { Polietileno com tratamento } \\
\text { UV }\end{array}$ \\
\hline
\end{tabular}

A vazão no tubo foi determinada por um medidor magnético indutivo de 1”, calibrado, com precisão de $0,5 \%$ instalado no início da tubulação. No final do tubo de polietileno foi instalado, sequencialmente, um tubo flexível de
1”, válvula reguladora de pressão e um aspersor. A válvula reguladora foi responsável por manter constante a pressão na base do aspersor e, consequentemente, assegurar descarga constante. Variando o bocal do 
aspersor foi possível ensaiar vazões equivalentes às velocidades de aproximadamente 1,$0 ; 1,5$ e $2,0 \mathrm{~m} \mathrm{~s}^{-1}$. Para cada vazão, avaliaram-se 3 amostras do tubo de polietileno, sob 9 pressões de operação. Após a obtenção da perda de carga contínua experimental, efetuaram-se cálculos para determinação deste mesmo parâmetro empregando as equações recomendadas pela literatura sendo: a) equação de Manning com fator de atrito $n$ igual a 0,008 (GOMES, 1997); b) equação de Fair-Whipple-Hsiao (NBR 5626, 1996); c) equação de Scobey com fator de atrito Ks igual a 0,32 (GOMES, 1997). A Figura 1 ilustra a estrutura utilizada para os ensaios de perda de carga contínua nos tubos ensaiados.

Para a determinação da perda de carga contínua nos tubos utilizados, não foi possível utilizar conexões disponíveis no mercado, por serem instaladas no interior dos tubos aumentando, assim, a perda de carga. Portanto, utilizou-se a metodologia proposta por GOMES et al. (2010) que empregaram uma conexão para envolver o tubo de polietileno, sendo que se utilizou anel de silicone para realização da vedação entre a conexão e o tubo (Figura 1C).

Para quantificar a perda de carga utilizouse um manômetro diferencial em " $U$ " com coluna de vidro e escala com precisão de mm (1000 - 0 - 1000) e líquido manométrico com massa específica $13600 \mathrm{~kg} \mathrm{~m}^{-3}$.

A perda contínua de carga foi determinada em segmentos de tubos de polietilenos de 40 metros retirados de uma bobina com 500 metros, sendo a primeira amostra retirada da parte inicial, a segunda da parte central e a terceira da parte final da mesma bobina. Os tubos ensaiados foram dispostos no laboratório de forma que ficassem em nível em todo o seu comprimento.

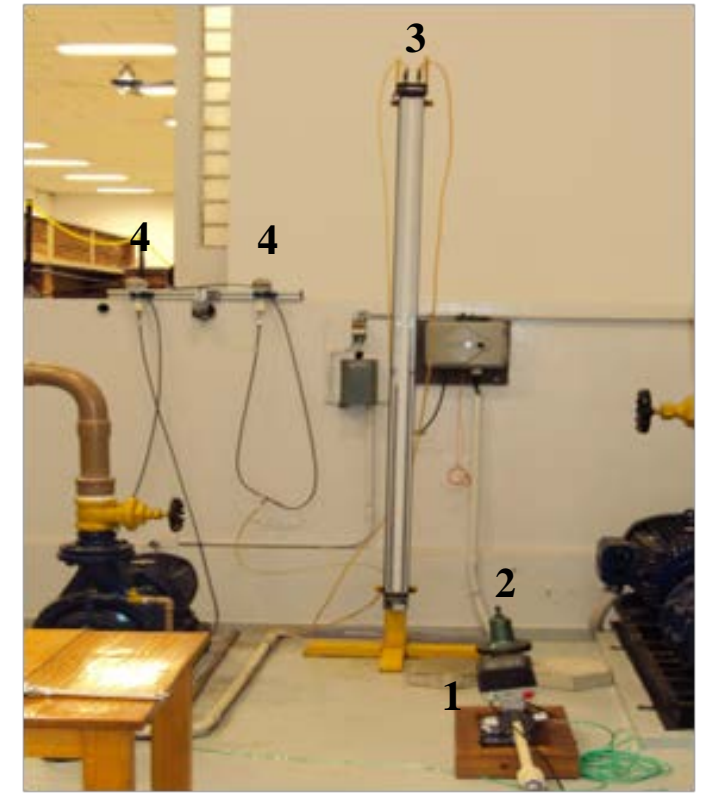

(A)

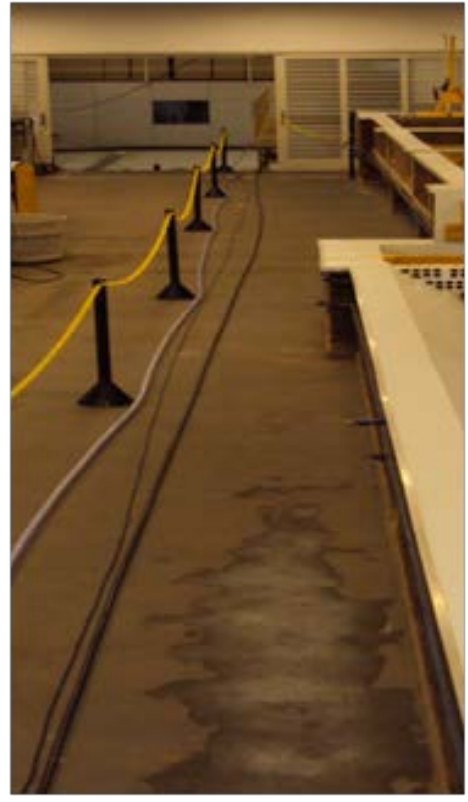

(B)

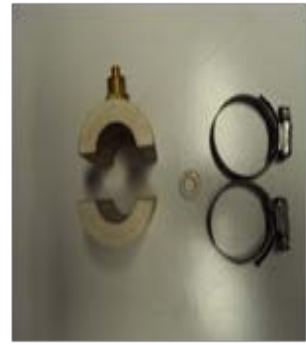

(C)

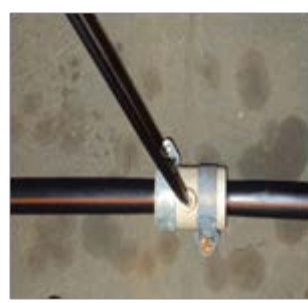

(D)

Figura 1. Estrutura utilizada no ensaio (A). Equipamentos: 1 medidor de vazão, 2 válvula, 3 manômetro diferencial e 4 manômetro digital; (B) Tubo de polietileno, (C) Conexão de PVC, (D) Conexão acoplada.

Para o monitoramento da pressão no tubo foram utilizados dois manômetros digitais calibrados, com precisão de $0,25 \%$, instalados no início e no final do tubo.

Construiu-se uma bancada de ensaio para pressurização em regime estático do fluido, que facilitou o controle, monitoramento e aquisição dos dados necessários para desenvolvimento do trabalho (Figura 2A).

O diâmetro externo dos tubos pressurizados, em regime estático, foi determinado empregando-se um micrômetro de varredura a laser, modelo LSM 503S (Figura 2A). Para determinação da alteração do 
diâmetro, as amostras de tubo foram pressurizadas de 0,0 a $588,4 \mathrm{kPa}$, com incrementos de 49,05 $\mathrm{kPa}$, totalizando 12 pontos de medição. Para cada amostra de tubo pressurizada foram efetuadas três leituras, sendo a primeira realizada a $0,25 \mathrm{~m}$ da extremidade $\left(L_{1}\right)$, a segunda a $0,5 \mathrm{~m}\left(\mathrm{~L}_{2}\right)$ e a terceira a $0,75 \mathrm{~m}\left(\mathrm{~L}_{3}\right)$. Devido ás amostras dos tubos não serem perfeitamente circulares foram realizadas medidas com o "scanner" na posição horizontal $\left(D_{1}\right)$ e na posição vertical $\left(\mathrm{D}_{2}\right)$. A Figura 2B ilustra os procedimentos utilizados.

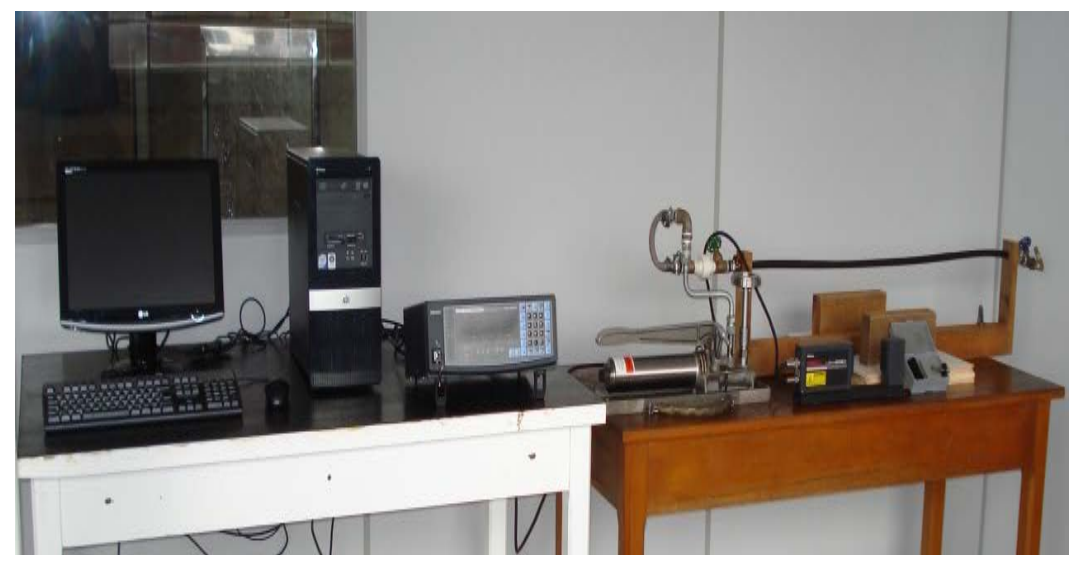

(A)
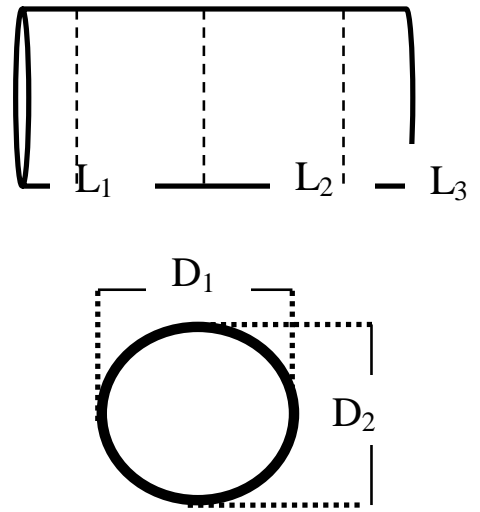

(B)

Figura 2. Bancada de ensaio (A) e Esquema de leitura do "scanner" a laser (B).

\section{RESULTADOS E DISCUSSÃO}

A vazão média e o coeficiente de variação de vazão para cada amostra, resultante das nove pressões avaliadas em 3 velocidades médias de escoamento, estão apresentados na (Tabela 2).
O coeficiente de variação reflete, em parte, a imperfeição do mecanismo de operação da válvula reguladora de pressão e consequentemente instabilidade na vazão, sendo todos os valores obtidos considerados aceitáveis.

Tabela 2. Vazão média e coeficiente de variação de vazão, resultante das nove pressões utilizadas, para as 3 amostras avaliadas e 3 velocidades médias

\begin{tabular}{|c|c|c|c|c|c|c|}
\hline \multirow{3}{*}{ Amostra } & \multicolumn{6}{|c|}{ Velocidade média $\left(\mathrm{m} \mathrm{s}^{-1}\right)$} \\
\hline & \multicolumn{2}{|c|}{1,06} & \multicolumn{2}{|l|}{1,57} & \multicolumn{2}{|l|}{1,87} \\
\hline & Vazão $\left(\mathrm{m}^{3} \mathrm{~h}^{-1}\right)$ & CV (\%) & Vazão $\left(\mathrm{m}^{3} \mathrm{~h}^{-1}\right)$ & $\mathrm{CV}(\%)$ & Vazão $\left(\mathrm{m}^{3} \mathrm{~h}^{-1}\right)$ & CV $(\%)$ \\
\hline 1 & 0,751 & 1,69 & 1,115 & 1,93 & 1,332 & 1,07 \\
\hline 2 & 0,749 & 2,80 & 1,101 & 1,65 & 1,312 & 1,68 \\
\hline 3 & 0,739 & 1,10 & 1,094 & 0,37 & 1,300 & 0,90 \\
\hline
\end{tabular}

Na Figura 3 apresenta a perda de carga contínua observada em laboratório versus a perda de carga contínua estimada pelas equações de Manning, Fair-Whipple-Hsiao e Scobey, considerando: para a velocidade de $1,06 \mathrm{~m} \mathrm{~s}^{-1} 10$ pressões de ensaio (147,1 a 588,4
kPa com incremento de 49,05 kPa), já para as vazões correspondentes as velocidades de 1,57 e $1,87 \mathrm{~m} \mathrm{~s}^{-1}$ foram utilizadas 9 pressões de ensaios que variaram de 196,2 $\mathrm{kPa}$ à 588,4 $\mathrm{kPa}$ com incremento de 49,05 $\mathrm{kPa}$. 

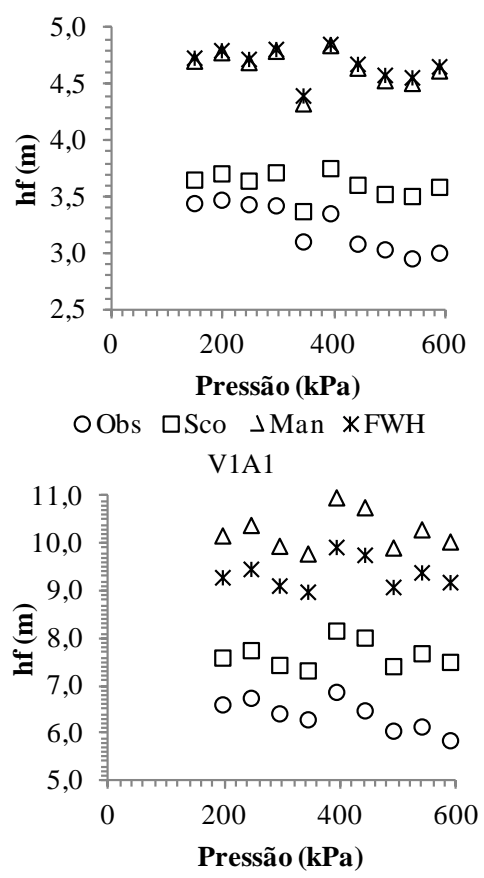

OObs $\square$ Sco $\rightarrow$ Man $*$ FWH V2A1

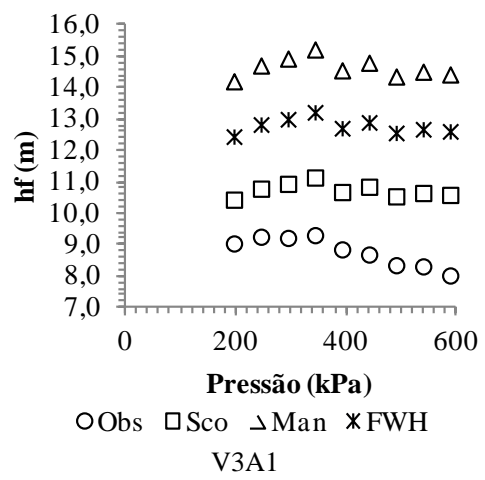

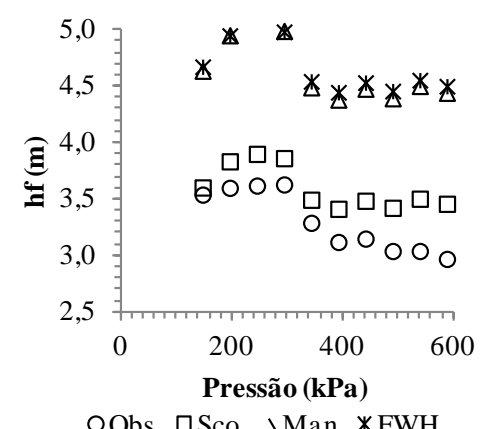

OObs $\square$ Sco $\lrcorner$ Man $*$ FWH

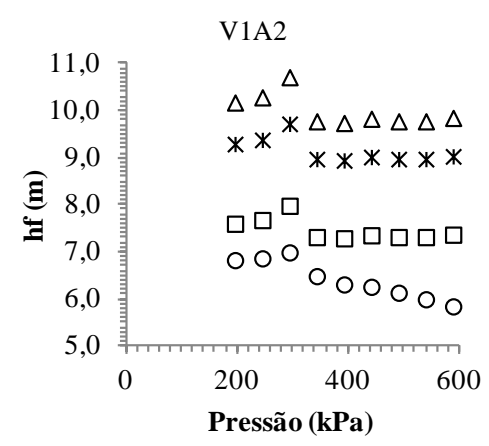

OObs $\square$ Sco $\lrcorner$ Man $*$ FWH V2A2

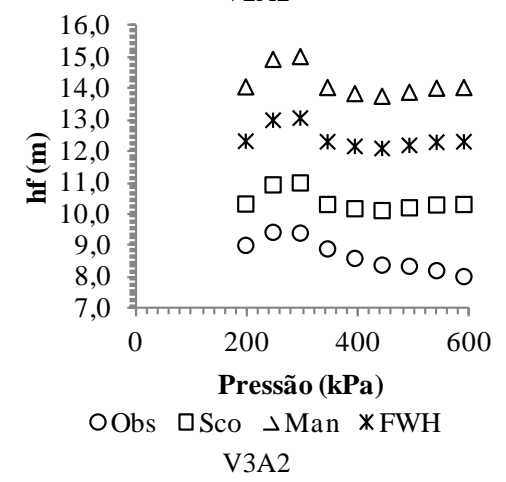

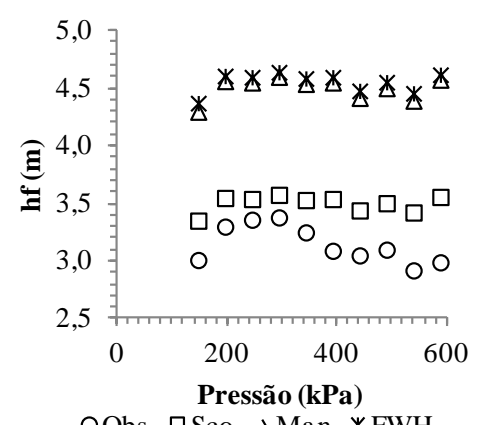

OObs $\square$ Sco $\triangle$ Man $*$ FWH

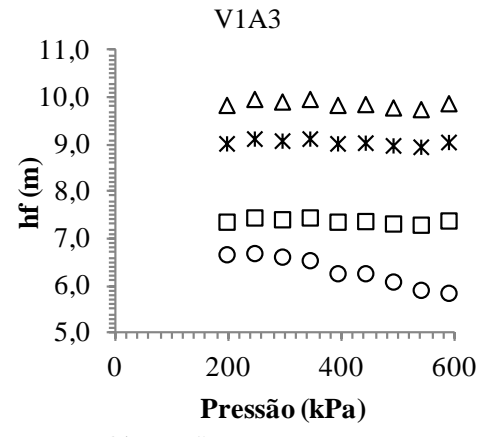

OObs $\mathrm{QSco} \rightarrow \mathrm{Man}$ *FWH V2A3

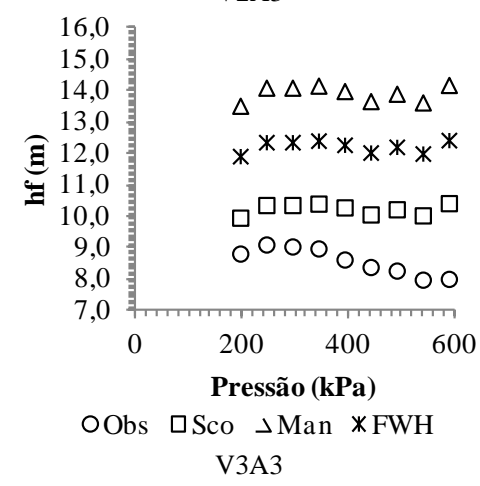

Figura 3.Representação gráfica da perda de carga contínua observada (Obs) e calculada pelas equações de Manning (Man), Fair-Whipple-Hsiao (FWH) e Scobey (Sco), em função da pressão, para as 3 amostras (A1, A2 e A3) e nas 3 velocidades de referência (V1=1,06 $\mathrm{m} \mathrm{s}^{-1}$; V2=1,57 $\mathrm{m} \mathrm{s}^{-1}$ e V3=1,87 $\left.\mathrm{m} \mathrm{s}^{-1}\right)$.

Em todas as condições avaliadas, as equações de Manning, Fair-Whipple-Hsiao e Scobey superestimaram a perda de carga contínua, quando aplicadas ao cálculo desta variável em tubos de polietileno. Houve redução na perda de carga, com o aumento da pressão de funcionamento, resultando em elevação do erro das equações em relação aos dados obtidos na determinação da perda de carga.

A superestimativa da perda de carga ocorre devido ao aumento do diâmetro dos tubos de polietileno com a elevação da pressão de operação. Vilela et al. (2003) ao avaliarem tubos de polietileno, com espessuras de paredes de 1325 e $1050 \mu \mathrm{m}$, observaram influência significativa da pressão de operação no diâmetro dos tubos e relataram que estas alterações podem ocasionar variações superiores a 20 \% na determinação da perda de carga.

Na Tabela 3 apresenta a variação do diâmetro externo do tubo de polietileno com a variação da pressão.

Tabela 3. Diâmetros observados em função da pressão de entrada

Pressão $(\mathrm{kPa}) \quad$ Diâmetro $(\mathrm{mm}) \quad$ Pressão $(\mathrm{kPa})$
Diâmetro (mm) 


\begin{tabular}{llll}
\hline 0,0 & 17,64 & 343,2 & 17,94 \\
98,1 & 17,73 & 392,3 & 17,98 \\
147,1 & 17,77 & 441,3 & 18,02 \\
196,1 & 17,81 & 490,4 & 18,06 \\
245,2 & 17,85 & 539,4 & 18,10 \\
294,2 & 17,89 & 588,4 & 18,14 \\
\hline
\end{tabular}

Na Tabela 4 apresenta o erro médio de estimativa das equações de Manning, FairWhipple-Hsiao e Scobey, quando utilizadas para calcular a perda de carga contínua em tubo de polietileno. Observou-se que para a faixa de pressão de $147,1 \mathrm{kPa}$ à $588,4 \mathrm{kPa}$ as estimativas foram superiores aos valores observados, sendo que, com o aumento da pressão o erro também aumentou. $\mathrm{O}$ erro na estimativa na perda de carga utilizando a equação de Manning variou de 36,5\% a $77,3 \%$ enquanto que a equação FairWhipple-Hsiao variou de 37,9\% a 55,3\%, já a equação de Scobey variou de $6,1 \%$ a $30,2 \%$. Assim, verifica-se que para tubos de polietileno a pressão afeta a determinação da perda de carga contínua.

Tabela 4. Erros médios (\%) na determinação da perda de carga em tubos de polietileno, devido ao incremento na pressão de funcionamento, utilizando a Equação de Manning (Man), FairWhipple-Hsiao (FWH) e Scobey (Sco).

\begin{tabular}{llllllllll}
\hline Pressão & \multicolumn{3}{c}{ Velocidade de 1,06 $\mathrm{m} \mathrm{s}^{-1}$} & \multicolumn{3}{c}{ Velocidade de 1,56 $\mathrm{m} \mathrm{s}^{-1}$} & \multicolumn{3}{c}{ Velocidade de 1,87 m s } \\
$(\mathrm{kPa})$ & Man & FWH & Sco & Man & FWH & Sco & Man & FWH & Sco \\
\hline 147,1 & 36,5 & 37,9 & 6,1 & ---- & ---- & ---- & ---- & ---- & ---- \\
196,1 & 37,8 & 38,4 & 6,9 & 50,1 & 37,3 & 12,2 & 55,5 & 36,5 & 14,3 \\
245,2 & 37,2 & 37,8 & 6,4 & 50,9 & 37,7 & 12,7 & 57,4 & 37,4 & 15,5 \\
294,2 & 37,9 & 38,3 & 6,9 & 52,6 & 39,4 & 14,0 & 59,2 & 38,9 & 16,8 \\
343,2 & 38,5 & 40,3 & 7,8 & 52,8 & 40,1 & 14,3 & 59,7 & 39,6 & 17,2 \\
392,3 & 44,0 & 45,3 & 12,0 & 57,1 & 43,4 & 17,3 & 62,5 & 42,5 & 19,4 \\
441,3 & 45,8 & 47,4 & 13,4 & 60,2 & 46,3 & 19,6 & 65,8 & 45,4 & 21,9 \\
490,4 & 46,4 & 48,1 & 13,9 & 61,2 & 47,9 & 20,6 & 68,8 & 48,1 & 24,1 \\
539,4 & 50,4 & 52,2 & 17,0 & 65,2 & 51,3 & 23,5 & 72,0 & 50,9 & 26,4 \\
588,4 & 52,1 & 53,6 & 18,3 & 69,6 & 55,4 & 26,8 & 77,3 & 55,3 & 30,2 \\
\hline
\end{tabular}

\section{CONCLUSÕES}

O erro na estimativa na perda de carga utilizando a equação de Manning variou de $36,5 \%$ a $77,3 \%$, já para a equação FairWhipple-Hsio a variação foi de 37,9\% a 55,3\%, para a equação de Scobey observou-se a variação entre $6,1 \%$ a $30,2 \%$.

A pressão de operação afetou a perda de carga contínua no tubo de polietileno estudado.

O polietileno apresentou variação de diâmetro quando submetido às diferentes pressões, caracterizado um comportamento elástico.

\section{AGRADECIMENTOS}

Ao Ministério da Ciência e Tecnologia (MCT), ao Conselho Nacional de Desenvolvimento Científico e Tecnológico (CNPq), à Fundação de Amparo à pesquisa do Estado de São Paulo (FAPESP), pelo apoio financeiro a esta pesquisa, através do Instituto Nacional de Ciência e Tecnologia em Engenharia da Irrigação (INCTEI). À Plasnova Louveira Indústria e Comércio Ltda.

\section{REFERÊNCIAS BIBLIOGRÁFICAS}


AZEVEDO NETTO, J. M.; ALVAREZ, G. A. Manual de hidráulica. 7 ed. São Paulo: Edgard Blücher, 1982, v1, 336p.

GOMES, H.P. Engenharia de irrigação. 2.ed. Campina Grande: UFPB,1997. 390 p.

GOMES, A. W. A; FRIZZONE, J.A; RETTORE NETO, O; MIRANDA, J. H. Perda de carga localizada em gotejadores integrados em tubos de polietileno. Engenharia Agrícola, Jaboticabal, v.30, n.3, p.435-446, 2010.

ASSOCIAÇÃO BRASILEIRA DE NORMAS TÉCNICAS. NBR 5626: Instalação Predial de Água Fria. Rio de Janeiro, 1996. 41 p.
PORTO, R. M. Hidráulica Básica. 4 ed. São Carlos: EESC-USP, 2006. 540 p.

RETTORE NETO, O. Modelo para determinação da perda de carga contínua em tubos elásticos. 2011.95 f. Tese (Doutorado em Irrigação e Drenagem) Universidade de São Paulo, Escola Superior de Agricultura Luiz de Queiroz, Piracicaba, 2011.

VILELA, L.A.A.; SOCCOL, O.J.; GERVÁZIO, E.S.; FRIZZONE, J.A.; BOTREL, T.A. Alteração no diâmetro de tubos de polietileno submetidos a diferentes pressões. Revista Brasileira de Engenharia Agrícola e Ambiental, Campina Grande, v.7, n.1, p.182185, 